\title{
On the quiescence of the Hubble flow in the vicinity of the Local Group
}

\section{A study using galaxies with distances from the Cepheid $P L$-relation}

\author{
T. Ekholm ${ }^{1,3}$, Yu. Baryshev ${ }^{2}$, P. Teerikorpi ${ }^{3}$, M. O. Hanski ${ }^{3}$, and G. Paturel ${ }^{1}$ \\ 1 CRAL - Observatoire de Lyon, 69561 Saint Genis Laval Cedex, France \\ 2 Astronomical Institute, St. Petersburg State University, Staryj Petergoff, 198504, St. Petersburg, Russia \\ 3 Tuorla Observatory, 21500 Piikkiö, Finland
}

Received 8 December 2000 / Accepted 31 January 2001

\begin{abstract}
Cepheid distances of local galaxies $(<7 \mathrm{Mpc})$ are used to study the very nearby velocity field, as pioneered by Sandage (1986) who also pointed out its remarkable properties: linearity and quietness. The new data show that the velocity dispersion in the distance range as seen from the barycentre of the Local Group $1-8 \mathrm{Mpc}$ is as low as $38 \mathrm{~km} \mathrm{~s}^{-1}$. The local rate of expansion coincides with the global Hubble constant. Down to $1.5 \mathrm{Mpc}$ we cannot detect a deviation from the linear Hubble flow. This puts an upper limit for the mass of the Local Group, for a wide class of Friedman models, including those with the cosmological constant.
\end{abstract}

Key words. cosmology: observations - galaxies: distances and redshifts - galaxies: general - galaxies: kinematics and dynamics - Local Group

\section{Introduction}

In his classical paper Sandage (1986) studied the perturbation of the very nearby velocity field using the available distances to local galaxies, mostly obtained by ground-based observations of brightest resolved stars and Cepheids. He concluded that the deviation from the linear Hubble flow possibly was detected at very small distances, while the quiet, linear Hubble law starts at about $2 \mathrm{Mpc}$. He pointed out the remarkable properties of the local velocity field: 1) The linearity of the velocity-distance relation down to small distances, 2) the closeness of the local and global rates of expansion, and 3) the small velocity dispersion around the Hubble law. He also asked what happens when the distances become more accurate, in particular, will the velocity dispersion still decrease below $\sigma_{\mathrm{v}}=60 \mathrm{~km} \mathrm{~s}^{-1}$ which he derived from those data. We also note that Karachentsev \& Makarov (1996) found that by using both massive and small galaxies the velocity dispersion in the local volume is $\sigma_{\mathrm{v}}=72 \mathrm{~km} \mathrm{~s}^{-1}$.

The aim of our Letter is to use new accurate data on the distances of nearby galaxies inferred for the extragalactic Cepheid $P L$-relation (cf. Sect. 2) in order to study further the behaviour of the Hubble law in the outskirts of the Local Group.

Send offprint requests to: T. Ekholm, e-mail: timo@typhon.univ-lyon1.fr

\section{The sample}

We make use of the the Lyon Extragalactic Cepheid Database (Lanoix et al. 1999b). In this database there are 32 galaxies with both $V$ and $I$ band photometry. 23 galaxies have Cepheid measurement from the HST and rest have groundbased measurements. The $P L$ relation was calibrated using a sample of galactic Cepheids measured with the astrometric HIPPARCOS satellite (Lanoix et al. 1999a) and corrected for a Malmquist-like bias according to Lanoix et al. (1999c).

As we are interested in the very local velocity field we restricted our sample by requiring $R_{\text {gal }} \leq 7 \mathrm{Mpc}$, where also the Virgo-centric flow correction is small. $R_{\text {gal }}$ refers to the distance in Mpc measured from Galaxy. We were left with 14 galaxies. The Virgo flow at larger distances was studied with Cepheid-based distances in Ekholm et al. (1999a).

In Table 1 we present the data relevant for the present analysis. In Col. 1 we give the PGC number and in Col. 2 we give the name. In Col. 3 we give the distance in $\mathrm{Mpc}$ as calculated from the distance moduli provided in the Lyon Extragalactic Cepheid Database. In Col. 4 we give the distance in $\mathrm{Mpc}$ as seen from the barycentre of the Local Group. In Cols. 5-7 we give the velocities: $V_{\odot}$ is the mean heliocentric velocity extracted from LEDA (the Lyon-Meudon Extragalactic Database), $V_{\text {Yahil }}$ is $V_{\odot}$ 
Table 1. In this table we present the the crucial data for the 14 galaxies with extragalactic $P L$-distances. The entries are explained in the text

\begin{tabular}{llccccc}
\hline LEDA & NAME & $R_{\text {gal }}$ & $R_{2 / 3}$ & $V_{\odot}$ & $V_{\text {Yahil }}$ & $V_{\text {corr }}$ \\
\hline 3844 & IC 1613 & 0.69 & 0.44 & -231 & -62 & -72 \\
45314 & IC 4182 & 4.94 & 5.09 & 316 & 337 & 274 \\
17223 & LMC & 0.05 & 0.61 & 317 & 81 & 82 \\
2557 & N224 & 0.87 & 0.29 & -300 & -13 & -12.7 \\
3238 & N300 & 2.17 & 2.13 & 142 & 125 & 101 \\
28630 & N3031 & 3.37 & 3.18 & -35 & 125 & 147 \\
29128 & N3109 & 1.02 & 1.53 & 404 & 130 & 131 \\
34554 & N3621 & 6.61 & 7.16 & 727 & 436 & 457 \\
48334 & N5253 & 3.16 & 3.73 & 403 & 155 & 153 \\
50063 & N5457 & 6.92 & 6.87 & 240 & 361 & 374 \\
5818 & N598 & 0.79 & 0.27 & -180 & 69 & 66 \\
63616 & N6822 & 0.45 & 0.73 & -56 & 8 & 11 \\
29653 & SEXA & 1.45 & 1.88 & 325 & 118 & 110 \\
28913 & SEXB & 1.39 & 1.76 & 302 & 139 & 131 \\
\hline
\end{tabular}

corrected for the centroid of the Local Group according to Yahil (1977) and $V_{\text {corr }}$ is $V_{\text {Yahil }}$ corrected for the virgocentric motion. We explain these corrections in the next section. As regards uncertainties in the parameters we note that the mean error for $V_{\odot}$ is $6.4 \mathrm{~km} \mathrm{~s}^{-1}$. In Fig. 1 we plot the error bars reflecting the uncertainties in the Cepheid distances.

Finally we remind that LMC and NGC 6822 belong to the Milky Way subsystem and NGC 598 (M 33) is a member of the M 31 subsystem. The status of the irregular galaxy IC 1613 is unclear. Gurzadian et al. (1993) and Rauzy \& Gurzadian (1998) link it with the Galaxy, but van den Bergh (1999) recognizes that IC 1613 may be a free-floating member of the Local Group. Thus it is wise to consider it as a special case in our diagrams.

\section{The method}

To study the local Hubble flow at smaller distances, it becomes increasingly important to make relevant corrections to distances and radial velocities. The first correction, discussed by Sandage (1986), is due to the shift of the observer to the centre of expansion, which in the selfgravitating Local Group is not in our Galaxy, but presumably in the barycentre. By assuming the mass ratio of M 31 and Galaxy is $M_{\mathrm{M} 31} / M_{\mathrm{G}} \approx 2$ Sandage set the barycentre to be on the line between M 31 and Galaxy at $2 / 3$ of the distance to M 31 . This is also our choice. We denote the distance from the barycentre by $R_{2 / 3}$.

The observed mean heliocentric velocities must also be corrected. We first correct the observed velocity to the value as it would be measured by an observer in our Galaxy being at rest relative to the centroid of the Local
Group $^{1}$. This we do according to Yahil et al. (1977), which is also the preferred choice in the LEDA database. We also remind that in Ekholm et al. (1999a) the correction was also made according to Richter et al. (1987) but difference to the correction of Yahil et al. (1977) was quite small. The velocity correction used in reads:

$\Delta v=295.4 \sin l \cos b-79.1 \cos l \cos b-37.6 \sin b$.

After the solar motion is thus corrected for, the infall to the Virgo Supercluster is the next disturbance. We correct the radial velocity for the virgocentric motion following Ekholm et al. (1999a):

$$
\begin{aligned}
V_{\text {corr }}=V_{\text {Yahil }} & \pm\left[v(d)_{\mathrm{H}}-v(d)\right] \\
& \times \sqrt{1-\sin ^{2} \Theta / d^{2}}+V_{\mathrm{LG}}^{\mathrm{in}} \cos \Theta,
\end{aligned}
$$

where $(-)$ is valid for points closer than the tangential point $d_{\text {gal }}<\cos \Theta$ and $(+)$ for $d_{\text {gal }} \geq \cos \Theta$.

$\Theta$ is the angular distance of a galaxy from the centre of Virgo and $d$ is its distance $R$ from the centre normalized to the distance of Virgo $\left(d=R / R_{\text {Virgo }}\right)$. Following Ekholm et al. (1999a) we take $R_{\text {Virgo }}=21 \mathrm{Mpc}$ and $H_{0}=57 \mathrm{~km} \mathrm{~s}^{-1} \mathrm{Mpc}^{-1}$. The Hubble velocity at a distance $d$ from the centre of Virgo is $v(d)_{\mathrm{H}}=V_{\text {cosm }}(1) \times d$. The cosmological velocity of the Virgo cluster becomes $V_{\text {cosm }}(1)=1200 \mathrm{~km} \mathrm{~s}^{-1}$. The predicted velocity $v(d)$ is solved using the Tolman-Bondi model as described by Ekholm et al. (1999a).

\section{Results}

We present the corrected velocity vs. distance relations for the very local velocity field $\left(R_{2 / 3}<8 \mathrm{Mpc}\right)$ in Figs. 1 and 2 . In Fig. 1 velocities are corrected only for the solar motion according to Yahil et al. (1977). We calculate the velocity dispersion without the Local Group members (the first 4 symbols marked as crosses), where certainly the internal dynamics masks the possible Hubble term which is small compared with the value of the velocity dispersion. The velocity dispersion is $\sigma_{\mathrm{v}}=42 \mathrm{~km} \mathrm{~s}^{-1}$ solved from galaxies between $R_{2 / 3} \in[1.0,8.0] \mathrm{Mpc}$. The expected Hubble law is given as a solid straight line and the $1 \sigma$ dispersion as dotted lines.

In Fig. 2 we show the influence of the correction for the virgocentric motion. Now the velocity dispersion decreases

\footnotetext{
1 We note that the observer at the centroid would measure a slightly different velocity, depending on the location of the galaxy and the nature of the velocity field close to the Local Group. For example, if the deviation of the velocity field from the Hubble flow is exactly spherically symmetric relative to the centroid, one may show by starting from the first principles of the Hubble velocity field that then the observer at the centroid would see the velocity $V_{\text {Yahil }} / \cos \theta . \theta$ is the angle between the centroid and the Galaxy as seen from the measured galaxy. In the present case, $\theta$ is normally quite small. Hence such a correction makes the velocity usually only $1-5 \mathrm{~km} \mathrm{~s}^{-1}$ larger for our field galaxies. We prefer here to use the simpler velocity correction as done by Sandage (1986).
} 
down to $\sigma_{\mathrm{v}}=37 \mathrm{~km} \mathrm{~s}^{-1}$. The decrease in $\sigma_{\mathrm{v}}$ is not significant. This is expected because most of the galaxies are at large angular distance from the Virgo except IC 4182 having $\Theta=26.4^{\circ}$. It is quite remarkable that after the correction this galaxy follows almost exactly the expected Hubble law.

Both Figs. 1 and 2 support the conclusion 4 of Sandage (1986). The observed random velocities get smaller as the uncertainties in the adopted distance indicator diminish.

Figure 3 shows the predictions for the velocity perturbations in Sandage's point-mass model for the case when the mass of the Local Group $M_{\mathrm{LG}}=210^{12} M_{\odot}$. For example, van den Bergh (1999) estimates that $M_{\mathrm{LG}}=$ $2.3 \pm 0.610^{12} M_{\odot}$. Evans et al. (2000) give an upper limit $M_{\mathrm{LG}}=2.410^{12} M_{\odot}$.

The velocities were deduced for three cosmologies using a numerical algorithm for the Tolman-Bondi calculations (Hanski et al. 2000). The solid curve shows the prediction for the now preferred Friedman model $\Omega_{\mathrm{m}}=0.3$ and $\Omega_{\Lambda}=0.7$. The dashed line corresponds to the classical flat model $\Omega_{\mathrm{m}}=1.0$ and $\Omega_{\Lambda}=0.0$. The dotted line is the prediction for a low mass-density universe $\Omega_{\mathrm{m}}=0.1$ and $\Omega_{\Lambda}=0.0 . \Omega_{\mathrm{m}}$ is the mean density parameter of the matter and $\Omega_{\Lambda}$ is the density parameter induced by the $\Lambda$-term. One may note that these models differ little from each other, which shows that the value of the velocity deflection (and the zero-velocity distance) is essentially determined by the mass of the LG.

Only galaxy that shows any significant deviation from the expected Hubble law is IC 1613 but as pointed out in Sect. 2 its dynamical status is not well known. On the other hand M 31 deviates only little from the Hubble law (in fact it is within the $1 \sigma$ limit).

Sandage (1986) discussed the position of the barycentre. He pondered whether it would be possible to reduce the scatter in the Hubble diagram by "changing the origin of the distances to the centroid as well". We have checked the behaviour of the velocity dispersion $\sigma_{\mathrm{v}}$ as a function of the position of the barycentre between Galaxy and M 31 .

Recently Evans et al. (2000) claimed that the halo of M 31 is about as massive as the halo of Galaxy. In this case the centroid would be at the middle distance. Now, $\sigma_{\mathrm{v}}$ changes quite slowly in the region between $R_{1 / 2}$ and $R_{2 / 3}$. For example at $R_{1 / 2}$ the dispersion $\sigma_{\mathrm{v}}=44 \mathrm{~km} \mathrm{~s}^{-1}$ and at $R_{1 / 4}$ it is $\sigma_{\mathrm{v}}=49 \mathrm{~km} \mathrm{~s}^{-1}$. It is thus not possible to make any statistically relevant claims about the exact position of the barycentre. With a larger sample of field galaxies, the position of the barycentre (and hence the mass ratio $M_{\mathrm{M} 31} / M_{\text {Galaxy }}$ ) could perhaps be determined.

Finally we note that due to the lack of galaxies with Cepheid distances within $R_{2 / 3}=1 \mathrm{Mpc}$ and $R_{2 / 3}=$ $2 \mathrm{Mpc}$ it is not possible to determine the zero-velocity surface. It is though important to note that galaxies within $R_{2 / 3}=1 \mathrm{Mpc}$ show practically no deflection as compared to the theoretical curves shown in Fig. 3, except possibly the "free-floating" IC 1613, which could fit some theoretical curve with $M_{\mathrm{LG}}<10^{12} M_{\odot}$.

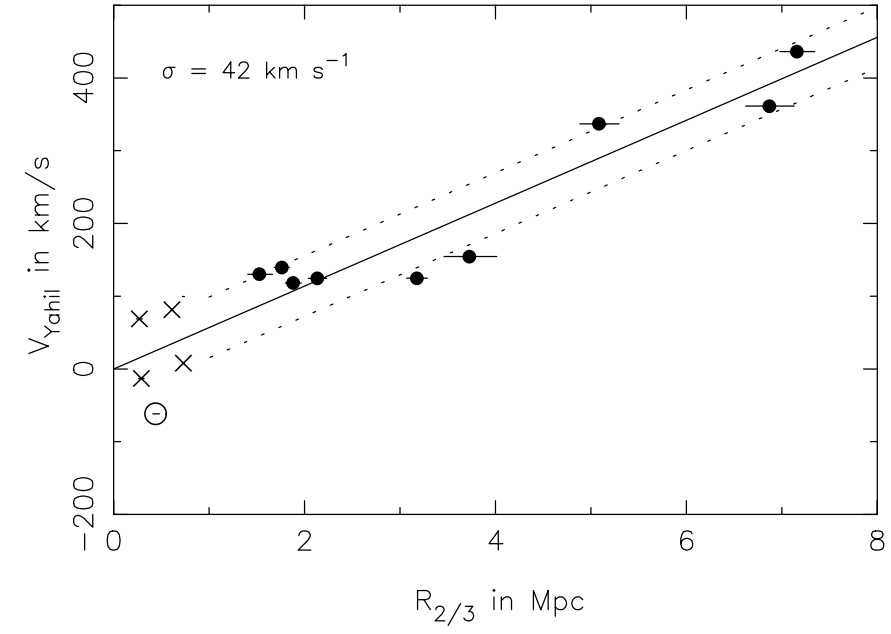

Fig. 1. Velocities corrected to the rest frame of the centroid of the Local Group according to Yahil et al. (1977). The origin of the distances has been changed to the centroid following Sandage (1986). The solid straight line is the Hubble law for $H_{0}=57 \mathrm{~km} \mathrm{~s}^{-1} \mathrm{Mpc}^{-1}$. The dotted lines give the $1 \sigma$ dispersion calculated for galaxies between $1 \mathrm{Mpc}$ and $8 \mathrm{Mpc}$ giving $\sigma=42 \mathrm{~km} \mathrm{~s}^{-1}$ Crosses refer to LMC, N6822 N224 and N598. The open circle is IC 1613. The error bars reflect the formal uncertainties in the Cepheid distances

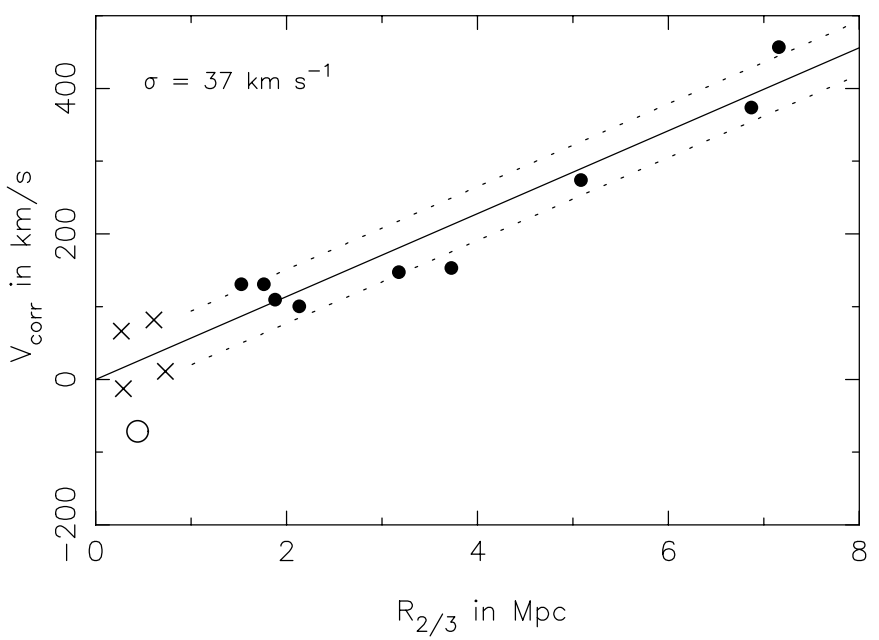

Fig. 2. As Fig. 1 except that now we have also corrected for the Virgocentric motions. The effect is small except for IC 4182. This is the only galaxy that is at a small angular distance from Virgo cluster. The velocity dispersion $\sigma_{\mathrm{v}}=37 \mathrm{~km} \mathrm{~s}^{-1}$

\section{Discussion and conclusions}

We may conclude that all the properties of the local Hubble flow, mentioned by Sandage, become even more remarkable when more accurate distances are used. Sandage (1986) noted that the derived velocity dispersion around the local Hubble law has progressively decreased together with increasing accuracy in distances, from Hubble's $200 \mathrm{~km} \mathrm{~s}^{-1}$ to his $60 \mathrm{~km} \mathrm{~s}^{-1}$. Our results confirm that this trend continues, and $\sigma_{\mathrm{v}} \leq 40 \mathrm{~km} \mathrm{~s}^{-1}$.

The velocity-distance diagrams (Figs. 2 and 3) show that the local Hubble constant is much the same as the 


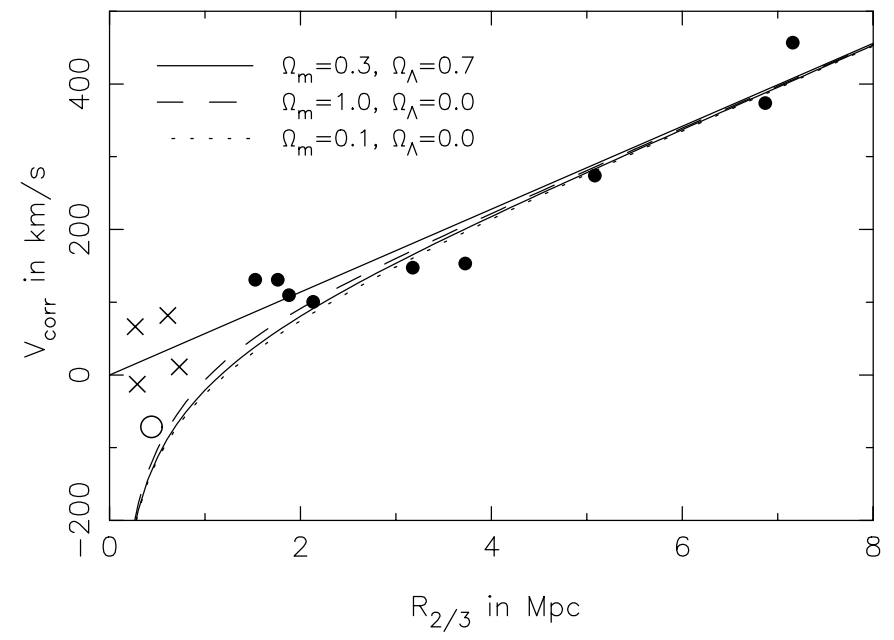

Fig. 3. As Fig. 2. Now we have added three theoretical predictions. Each of them is based on a point mass model assuming that the mass of the Local Group is $M_{\mathrm{LG}}=210^{12} M_{\odot}$. We examined three cosmologies: 1) the Friedman model with $\Omega_{\mathrm{m}}=0.3$ and $\Omega_{\Lambda}=0.7$ (the solid curve), 2) the classical flat model with $\Omega_{\mathrm{m}}=1.0$ and $\Omega_{\Lambda}=0.0$ (the dashed curve) and 3) a low mass density model with $\Omega_{\mathrm{m}}=0.1$ and $\Omega_{\Lambda}=0.0$ (the dotted curve)

more global value derived from the Tully-Fisher indicator from the KLUN sample (Theureau et al. 1997; Ekholm et al. 1999b). This also confirms Sandage's (1999) recent estimate that the very local Hubble constant is the same as the global $H_{0}$ within 10 percent.

The third important feature is the small distance where the Hubble law emerges. The new data show that the linear Hubble law extends down to at least 1.5 Mpc. Hence, within the standard Friedman model (including the cosmological constant $\Lambda$; see Fig. 3 ), one gets an upper limit for the mass of the Local Group $\left(\approx 210^{12} M_{\odot}\right)$.

The quiet Hubble flow within the very clumpy local galaxy universe has always been a real riddle (Sandage et al. 1972; Sandage 1999). The problem of the local quiet Hubble flow was studied by Governato et al. (1997) using high-resolution CDM $N$-body simulations. They constructed a large sample of "Local Groups" and calculated the velocity dispersions in $5 \mathrm{Mpc}$ volumes around the LG candidates. They found for $\Omega=1 \mathrm{CDM}$ model that the velocity dispersion is $300-700 \mathrm{~km} \mathrm{~s}^{-1}$ and for $\Omega=0.3 \mathrm{CDM}$ model $150-300 \mathrm{~km} \mathrm{~s}^{-1}$. They state that these simulations were unable to produce a single LG having a velocity dispersion as low as observed.

The very local Hubble diagram offers several important applications for cosmology when the number of accurate Cepheid distances to local galaxies is increased. The mass of the Local Group and the position of its barycenter may be determined more precisely. And recently it has been suggested (Chernin et al. 2000) that the quietness of the local Hubble flow is a signature of the cosmological vacuum (Einstein's $\Lambda$-constant corresponding to energy density $\rho_{\Lambda}=$ const.) dominated universe where the velocity perturbations are adiabatically decreasing. For solution of both problems - the small velocity dispersion and the Hubble law starting immediately at $1.5 \mathrm{Mpc}$ with the global $H_{0}$ - Baryshev et al. (2000) proposed that there is a cosmological, homogeneous dark energy (quintessence) component with time-variable energy density $\rho_{\mathrm{Q}}(t)$ and equation of state $p_{\mathrm{Q}}=w \rho_{\mathrm{Q}} c^{2}$ with $w \in[-1,0]$. These discussions show that the very local volume is extremely important for the study of global properties of the universe.

Acknowledgements. This work was partly supported by the Academy of Finland (project 45087: "Galaxy Streams and Structures in the nearby Universe" and project "Cosmology in the Local Galaxy Universe"). We have made use of the Lyon-Meudon Extragalactic Database LEDA and the Lyon Extragalactic Cepheid Database. We are grateful for the referee, M. Capaccioli, for his comments.

\section{References}

Baryshev, Yu., Chernin, A., \& Teerikorpi, P. 2000 [astro-ph/0011528]

Chernin, A., Teerikorpi, P., \& Baryshev, Yu. 2000, Adv. Space Res., in press [astro-ph/0012021]

Ekholm, T., Lanoix, P., Teerikorpi, P., et al. 1999a, A\&A, 351, 827

Ekholm, T., Teerikorpi, P., Theureau, G., et al. 1999b, A\&A, 347,99

Evans, N. W., Wilkinson, M. I., Guhathakurta, P., et al. 2000, ApJ, 540, L9

Governato, F., Moore, B., Cen, R., et al. 1997, New Astron., 2,91

Gurzadian, V. G., Kocharyan, A. A., \& Petrosian, A. R. 1993, Ap\&SS, 201, 243

Hanski, M. O., Theureau, G., Ekholm, T., \& Teerikorpi, P., A\&A, submitted

Karachentsev, I. D., \& Makarov, D. A. 1996, AJ, 111, 794

Lanoix, P., Paturel, G., \& Garnier, R. 1999a, MNRAS, 308, 969

Lanoix, P., Garnier, R., Paturel, G., et al. 1999b, Astron. Nachr., 320, 21

Lanoix, P., Paturel, G., \& Garnier, R. 1999c, ApJ, 516, 188

Sandage, A. 1986, ApJ, 307, 1

Sandage, A. 1999, ApJ, 527, 479

Sandage, A., Tammann, G., \& Hardy, E. 1972, ApJ, 172, 253

Rauzy, S., \& Gurzadian, V. G. 1998, MNRAS, 298, 114

Richter, O.-G., Tammann, G. A., \& Huchtmeier, W. K. 1987, $\mathrm{A} \& \mathrm{~A}, 171,33$

Theureau, G., Hanski, M., Ekholm, T., et al. 1997, A\&A, 322, 730

van den Bergh, S. 1999, AAR, 9, 273, 26

Yahil, A., Tammann G. A., \& Sandage, A. 1977, ApJ, 217, 903 